\title{
Erratum to: On Conjugate Times of LQ Optimal Control Problems
}

\author{
Andrei Agrachev • Luca Rizzi • Pavel Silveira
}

Published online: 10 February 2015

(C) Springer Science+Business Media New York 2015

\section{Erratum to: J Dyn Control Syst (2014)}

\section{DOI 10.1007/s10883-014-9251-6}

There is the following misprint in the paper.

Affiliation of Andrei Agrachev should read as follows:

(1) SISSA, Trieste, Italy

(2) IM SB RAS, Novosibirsk, Russia

The online version of the original article can be found at http://dx.doi.org/10.1007/s10883-014-9251-6.

A. Agrachev $(\triangle)$ · P. Silveira

SISSA, Trieste, Italy

e-mail: agrachev@sissa.it

A. Agrachev

IM SB RAS, Novosibirsk, Russia

L. Rizzi

CNRS, CMAP École Polytechnique and Équipe INRIA GECO Saclay Île-de-France, Paris, France 